Rev. Colombiana cienc. Anim. 4(2):410-434,2012

ORIGINAL

\title{
PALINOMORFOS DEL CUATERNARIO SOBRE EL VALLE DEL RIO SAN EUGENIO (RISARALDA, COLOMBIA)
}

\section{QUATERNARY PALYNOMORPH FROMTHE VALLEY OF THE RIVER SAN EUGENIO (RISARALDA, COLOMBIA)}

MERCADO, G., JORGE ${ }^{1}$ M.Sc, ACEITUNO, B. FRANCISCO ${ }^{2}$ Ph.D, PEREZ, C., ALEXANDER $^{3}$ Dr.

${ }^{1}$ Departamento de Biología, Facultad de Educación y Ciencias, Universidad de Sucre, Sincelejo, Colombia. ${ }^{2}$ Departamento de Antropología. Universidad de Antioquia, Apartado 1226, Medellín, Colombia. ${ }^{3}$ Universidad de Sucre, Facultad de Ciencias Agropecuarias, Grupo Bioprospección Agropecuaria.

Correspondencia: fjaceituno@quimbaya.udea.edu.co, jdmercadog@gmail.com

Recibido: 15 -04- 2012; Aceptado: 25-07-2012.

\section{Resumen}

Un análisis cualitativo de las observaciones palinológicas realizadas sobre un yacimiento arqueológico entre el Pleistoceno tardío hasta el Holoceno temprano sobre Santa rosa de Cabal, Risaralda Colombia fue llevado a cabo como parte de una reconstrucción paleoecológica, dentro de la cual se analizaron un total de 71 muestras a partir de dos núcleos suelos volcánicos, La pochola 01 (47 muestras) y la pochola 02 (25 muestras). Con base en los datos de ${ }^{14} \mathrm{C}$ obtenidos de la Pochola 01 se pudo establecer que la edad más antigua es de $13.540 \pm 60$ años A.P, en los $105 \mathrm{~cm}$ (Pleistoceno tardío) y la edad más reciente $6743 \pm 45$ años a.P en los 57 $\mathrm{cm}$, (Holoceno medio). Los resultados muestran que la preservación de los granos de polen en suelos volcánicos es alta,la diversidad y buena preservación. Un total de 67 especies fueron descritas dentro de las cuales se encuentran representantes de clases, Pteridophytos (11 especies), Pinopsida (1especies) y Angiospermas (50 especies).Mediante este documento se pretende incrementar y contribuir al conocimiento palinológico del Cuaternario en la región del Cauca Medio.

Palabras clave: palinología, cuaternario, Cauca medio, bosques subandinos.

\section{Abstract}

Quantitative analysis of the palynological observations in an archaeological settlement between Late Pleistocene to Early Holocene in Santa Rosa de Cabal, Risaralda Colombia. A total of 71 samples were analysed over volcanic soils $\mathrm{La}$ pochola01 (47 samples) and La pochola02 (25 samples). The radiocarbon result 
dated the oldest simple which is $13.540 \pm 60$ years B.P in the $105 \mathrm{~cm}$ (Late Pleistocene) and the earliest simple which was $6743 \pm 45$ years B.P in the $57 \mathrm{~cm}$, (Early Holocene). Diversified, well preserved palynofloras were recognized. A total of 61 species were described, including species of the, Pteridophytos (11 species), Pinopsida (1species) and Angiosperm (50species). This paper aims to contribute an increase to the knowledge of the Quaternary palynoflora in the middle Cauca.

Key word: palynology, quaternary, middle Cauca, subandean forest.

\section{Introducción}

Las reconstrucciones paleoambientales basadas en análisis palinológicos han sido importantes para entender el carácter dinámico de los ecosistemas naturales y la evolución de las poblaciones humanas que habitaron América sobre los últimos 10.000 años a.P (HOOGHIEMSTRA y VAN DER HAMMEN, 2004; HOOGHIEMSTRA et al., 2006). Colombia ha jugado un papel importante durante el proceso de colonización y expansión humana por el continente; dada su ubicación geográfica fue el principal corredor de paso para ingresar a Suramérica y por tanto de gran importancia para entender la colonización en la región andina, así como la evolución de los procesos de domesticación y manejo del bosque (ACEITUNO y LOAIZA, 2007). La región conocida como el Cauca medio, ha sido uno de los principales corredores de expansión humana a través del continente, lo que hace de esta región de gran importancia a la hora de estudiar el comportamiento de la vegetación, el clima y la interacción del hombre con el medio. Gran parte de los valles del Cauca Medio, sobre el Pleistoceno final y el Holoceno medio, estuvieron ocupados por comunidades humadas (cazadoresrecolectores), estas poblaciones manipularon su entorno para optimizar sus ocupaciones, manejando plantas autóctonas como Dioscoreasp.o Xanthosoma sp.y foráneas como el Zea mays o Manihot sp.(ACEITUNO y CASTILLO, 2005; ACEITUNO y LOAIZA, 2006).

La base fundamental para analizar los cambios en la estructura de diferentes ambientes boscosos sobre distintas latitudes geográficas, la dinámica climática de una región y la presión antrópica durante el Cuaternario han sido los análisis palinológicos aplicados a reconstrucciones paleoecológicas(BERRíO et al., 2001; PIPERNO y PEARSALL, 1998; WILLE et al., 2001; WILLE et al., 2000; ZARATE et al., 2005); ya que las plantas son susceptibles a las variaciones climáticas, debido a sus niveles de tolerancia; y a la alteración antrópica, dependiendo de la estrategia de explotación por parte del hombre. 
En este estudio se realiza una caracterización preliminar de la vegetación en las diferentes zonas de vida ubicadas en la Cuenca del rio San Eugenio, basados en diversos estudios e inventarios realizados por los autores. De igual forma se presenta la primera parte de los palinomorfos estudiados en dos perfiles arqueológicos sobre los sedimentos volcánicos de la cuenca del Rio san Eugenio, a través de descripciones palinológicas, ya que este lugar carece de catálogos, estudios 0 atlas palinológicos, que funcionen como una base o marco de referencia para mejorar la comprensión sobre la sensibilidad ecológica de los biomas andinos en el Cauca Medio, ante la influencia del hombre y el clima.

\section{Materiales y Métodos}

Área de estudio. El valle medio del río San Eugenio se encuentra en el municipio de Santa Rosa de Cabal, localizado en el piedemonte occidental de la Cordillera Central, en las coordenadas geográficas Latitud N $4^{\circ} 53^{\prime} 32.7^{\prime \prime}$ y Longitud W $75^{\circ}$ 38'01.9", en la región conocida como Cauca medio (Fig.1), dentro de los márgenes del bosque subandino ( 1.000 y $2.400 \mathrm{~m}$ ). Los suelos de la zona de estudio se han desarrollado fundamentalmente a partir de un sustrato metasedimentario volcánico de edad cretácica (sedimento de mar profundo y lavas interestratificadas). En términos edafológicos pueden ser clasificados como metasedimentarios con cenizas volcánicas, con elevados porcentajes de materia orgánica y a una alta capacidad de intercambio de cationes, que hacen de estos suelos altamente fértiles (CASCAREDO et al., 2001; CORTÉS, 1978; JARAMILLO et al., 1994; SALOMONS, 1986).

Su valor palinológico radica en los agregados de minerales, materia orgánica, restos orgánicos, vidrios volcánicos, minerales primarios y secundarios; estos elementos son cementados por el alófono (un producto amorfo emanado por el rápido y alto calentamiento de los vidrios volcánicos), el cual genera ácido húmico (de ahí su nombre alófono húmico) que recude el $\mathrm{pH}$ entre 5,2 y 6,1. (SALOMONS, 1986); estas características hacen de estos suelos óptimos para la preservación de los granos de polen y esporas.

Muestreo. Los sedimentos fueron colectados directamente del sitio arqueológico la Pochola 01 y un sitio adyacente la Pochola 02, a través de la técnica de canaletas, la cual tiene la ventaja de extraer el perfil completo. En el laboratorio se realizo el muestro a intervalos de $3 \mathrm{~cm}$ para el análisis de polen. Las muestras fueron procesadas de acuerdo al método estándar de extracción, el cual incluye un tratamiento $\mathrm{HCl}$, pirofosfato de sodio, $\mathrm{HF}$ y $\mathrm{KOH}$ y montaje en gelatina 
glicerinada. Para la identificación de los granos de polen y esporas fósiles fueron usados atlas polínicos (HERRERA y URREGO, 1996; HOOGHIEMSTRA, 1984; MERCADO-GÓMEZ, 2007; MERCADO-GÓMEZ et al., 2007; ROUBIK y MORENO, 1991) y las colecciones de referencia de las palinotecas de las Universidades de Antioquia y Pamplona.

Todos los granos de polen y esporas identificados en este trabajo son descritos e ilustrados en las fotoláminas. El sufijo "tipo" es usado debido a que los granos hallados en el registro pueden tener afinidad a varios grupos de plantas. Los procedimientos yabreviaturas son los mismos que los citados por MERCADOGÓMEZ, et al. (2011) para las descripciones de los polimorfos, siendo; Vista Ecuatorial: eje ecuatorial (E.E), eje polar (E.P), para el caso de los granos monoporados fue necesario utilizar abreviatura D.1, la cual es similar a D.E por dificultad al reconocer la polaridad. Las observaciones fueron realizadas en un equipo Olympus siguiendo la nomenclatura de FAEGRI e IVERSEN (1975) y PUNT et al(1994). Todas las medidas son dadas en micrones y en lo posible se contaron 10 granos por entidad palinológica. Los palinomorfos son presentados en orden evolutivo, desdePteridophytos, Pinopsida, Monocotiledoneas y Eucotiledoneas. Dentro de cada división las especies fueron arregladas en orden alfabético.
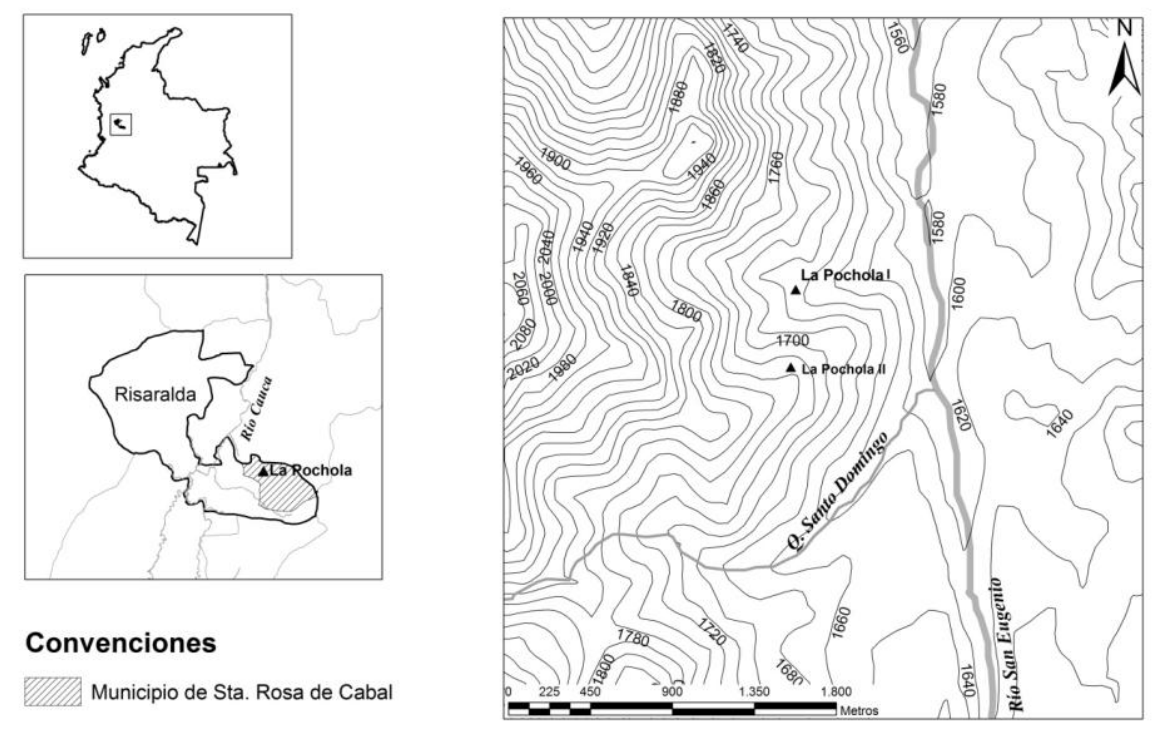

Figura 1. Cuenca del rio San Eugenio, mostrando los intervalos y curvasaltitudinales. A la derecha se observan la ubicación geográfica del área de estudio en Santa Rosa de Cabal Risaralda-Colombia y a la izquierda la ubicación de Pochola 01 y Pochola 02 
Para ubicar la formación del sitio en una escala temporal, se realizaron dataciones de ${ }^{14} \mathrm{C}$ por medio del AMS (accelerator mass spectrometer) y calibradas en años calendario usando el software OxCal Ver. 3.5, a partir de muestras de carbón vegetal asociadas a los pisos de ocupación vinculados a los horizontes de suelo. Estos análisis fueron llevados a cabo en el Centro di DAtazione e Diagnostica Dipartimento di Ingegneria dell'Innovazione, Università del Salento, Italia.

\section{Resultados}

Datos de radio carbono. Con base a los datos de radiocarbono del AMS del sitio la Pochola 01 (Fig. 2), fue posible identificar las edades relativas dentro de la columna analizada, así como un control cronológico de los sedimentos. Los resultados de ${ }^{14} \mathrm{C}$ indican que la edad más antigua data del $13.540 \pm 60$ años a.P, en los $105 \mathrm{~cm}$, correspondiendo al Pleistoceno tardío y la edad más reciente $6743 \pm 45$ años a.P en los $57 \mathrm{~cm}$, correspondiendo al Holoceno medio.

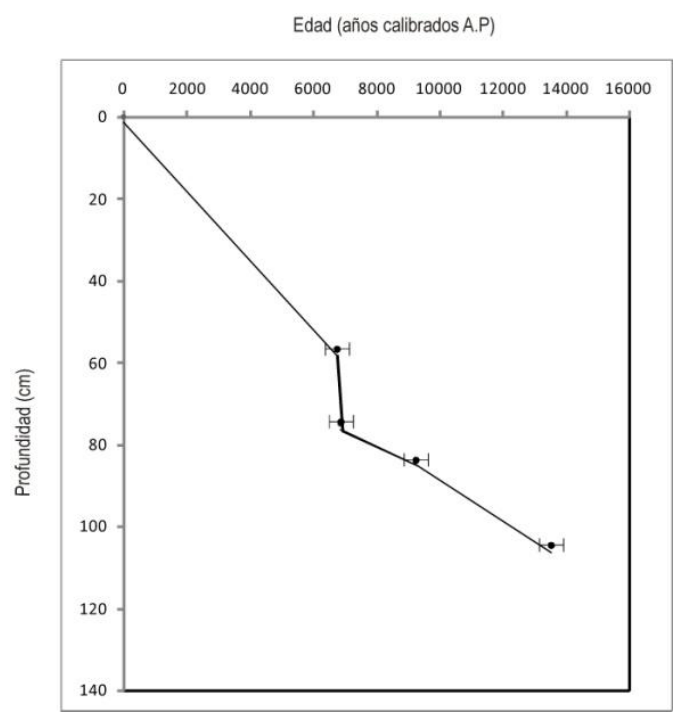

Figura 2. Cronología del registro la Pochola 01 , basado en los datos de ${ }^{14} \mathrm{C}$, en relación a la profundidad. Los cirulos negros representan el valor medio para cada edad relativa y las barras representan la desviación estándar

Descripción de los palinomorfosy edad del sitio. Fueron descritos entre esporas y granos de polen 62 especies, que incluyen: 11 especies de Pteridophytos, 1 especie de Pinopsida y 50 especies de Angiospermas.

\section{Pteridophytos}

\subsection{Familia Selaginellaceae}

TipoSelaginella (Lámina 1, Fig. 1-1a) 
Descripción: Espora, simétrica radial, heteropolar, ámbito triangular obtusoconvexo; trilete, labrum ausente, curvatura ausente, laesura extendiéndose al ecuador, ápices agudos, comisura recta; esporodermo de $1 \mu \mathrm{m}$ de grosor, escultura equinada en ambas caras, equinas de $\leq 1 \mu \mathrm{m}$ de largo por $0.5 \mu \mathrm{m}$ de ancho. Dimensiones: D.E $14 \mu \mathrm{m}$.

\subsection{Familia Dryopteridaceae}

Tipo Ctenitis (Lámina 1, Fig. 2-2a)

Descripción: Espora, simétrica bilateral, heteropolar, ámbito elíptico a circular; monolete, laesura de $22 \mu \mathrm{m}$ de largo por $1 \mu \mathrm{m}$ de ancho; esporodermo de una capa, de escultura psilada, perina presente con prominentes paredes ruguladas y espinadas. Dimensiones: D.1: $25 \mu \mathrm{m}$.

\subsection{Familia Polypodiaceae}

Tipo Grammitis (Lámina 1, Fig. 3-3a)

Descripción: Espora, radialmente simetría, ámbito circular; trilete, laesura alcanzando hasta el ecuador, labro de $3 \mu \mathrm{m}$ de grosor; comisura ligeramente ondulada, curvatura ausente; hacia la región inter-radial, se observa una depresión fuerte; esporodermo de una capa de escultura verrugada en ambas caras, verrugas escabradas de formas variadas y homogéneas sobre toda la superficie del grano. Dimensiones:D.E $35 \mu \mathrm{m}$.

Tipo Microgramma (Lámina 1, Fig. 4)

Descripción: Espora, lateralmente plano convexa, ámbito elíptico a circular; monolete, laesura de $30 \mu \mathrm{m}$ de largo por $2 \mu \mathrm{m}$ de ancho; esporodermo de una capa, escultura verrugada en la cara distal, verrugas heterogéneas dispuestas sobre toda el lado distal; mientras que la cara proximal es reticulada. Dimensiones: D.E $35 \mu \mathrm{m}$.

Tipo Polypodiaceae (Lámina 1, Fig. 5-5a)

Descripción: Espora, lateralmente plano convexa, ámbito elíptico a circular; monolete, laesura de $30 \mu \mathrm{m}$ de largo por $2 \mu \mathrm{m}$ de ancho; esporodermo de una capa, escultura verrugada en la cara distal, verrugas heterogéneas dispuestas sobre toda el lado distal; mientras que la cara proximal es reticulada. Dimensiones:D.E 42- 52 $\mu \mathrm{m}$.

Tipo Monoleteperinado (Lámina 1, Fig. 6)

Descripción: Espora, lateralmente plano convexa, heteropolar, ámbito elíptico a circular; monolete, comisura de $17 \mu \mathrm{m}$ de largo por $1 \mu \mathrm{m}$ de ancho, labro $\leq 1 \mu \mathrm{m}$ de 
borde ondulado; esporodermo de $3 \mu$ mde grosor, escultura reticulada, con lúmenes $\geq 2 \mu \mathrm{m}$. Dimensiones: D.E $14 \mu \mathrm{m}$.

\subsection{Familia Cyatheaceae}

Tipo Cyathea sp1 (Lámina 1, Fig. 7-7a)

Descripción: Espora, radialmente simétrica, heteropolar, ámbito triangular obtusoconvexo; trilete, laesura extendiéndose al ecuador, labro de $6 \mu \mathrm{m}$ de grosor, de borde ondulado, comisura ligeramente ondulada, curvatura ausente; esporodermo de $8 \mu \mathrm{m}$ de grosor, escultura rugulada a reticulada en la cara proximal; el lado distal es psilado. Dimensiones:D.E $44 \mu \mathrm{m}$.

Tipo Cyathea sp2 (Lámina 1, Fig. 8)

Descripción: Espora, radialmente simétrica, heteropolar, ámbito triangular obtusoconvexo; trilete, laesura extendiéndose hasta el ecuador; labro de $3 \mu \mathrm{m}$ de grosor; esporodermo $2 \mu$ mde grosor, escultura verrugada en ambas caras. Dimensiones:D.E $22 \mu \mathrm{m}$.

\subsection{Familia Dicksoniaceae}

Tipo Dicksonia (Lámina 1, Fig. 9-9a)

Descripción: Espora, radialmente simétrica, heteropolar, ámbito triangular obtusoconvexo; trilete, laesura con ápices agudos extendiéndose al ecuador, labro de $6 \mu \mathrm{m}$ de grosor de borde recto, comisura ligeramente ondulada y curvatura imperfecta; esporodermo de $8 \mu \mathrm{m}$ de grosor, escultura rugulada a reticulada en la cara proximal; mientras que la cara distal es pislada. Dimensiones: D.E $44 \mu \mathrm{m}$.

\subsection{Familia Lycopodiaceae}

Tipo Lycopodium (Lámina 1, Fig. 10-10a)

Descripción: Espora, radialmente simétrica, heteropolar, ámbito triangular obtusoconvexo; trilete, laesura con ápices agudos extendiéndose al ecuador; comisura ligeramente ondulada y curvatura ausente; esporodermo de $2.5 \mu \mathrm{m}$ de grosor, escultura reticulada en ambas caras. Dimensiones:D.E $44 \mu \mathrm{m}$.

\subsection{Familia Pteridaceae}

Tipo Jamesonia (Lámina 1, Fig. 11-11a)

Descripción: Espora, radialmente simétrica, heteropolar, ámbito triangular obtusoconvexo; trilete, laesura con ápices agudos extendiéndose al ecuador; labro de $6 \mu \mathrm{m}$ de grosor de borde recto; comisura ligeramente ondulada y curvatura ausente; esporodermo de $8 \mu \mathrm{m}$ de grosor, escultura rugulada en ambas caras. Dimensiones:D.E $44 \mu \mathrm{m}$. 


\section{Pinopsida}

\subsection{Familia Podocarpaceae}

Tipo Podocarpus (Lámina 2, Fig. 12-12a)

Descripción: Mónada, sacado; el cuerpo basal inaberturado de tamaño mediano a grande y escultura escabrada. Dimensiones: $40 \mu \mathrm{m}$ por $60 \mu \mathrm{m}$.

\section{Plantas con flores}

\section{Monocotiledonea}

\subsection{Familia Araceae}

Tipo Araceae (Lámina 2, Fig. 13)

Descripción: Mónada, apolar, ámbito esferoidal, tamaño del grano grande; inaberturado; exina tectada, escultura psilada. Dimensiones:D1 50-67 $\mu \mathrm{m}$.

TipoAnthurium (Lámina 2, Fig. 14-14a)

Descripción: Mónada, isopolar, simétrica radial, forma oblado esferoidal (P/E 1.07); inaberturadootriporado, poros de $1 \mu \mathrm{m}$; exina semitectada de $1 \mu \mathrm{m}$ de grosor, escultura reticulada, lúmenes heterobrochados, muro y lumen $\leq 1 \mu \mathrm{m}$. Dimensiones:E.P $15 \mu \mathrm{m}$; E.E $14 \mu \mathrm{m}$.

TipoPhilodendrum (Lámina 2, Fig. 15)

Descripción: Mónada, heteropolar, simétrica bilateral, ámbito elíptico; monosulcado, margen de $1 \mu \mathrm{m}$ de ancho; exina tectada de $1 \mu \mathrm{m}$ de grosor,

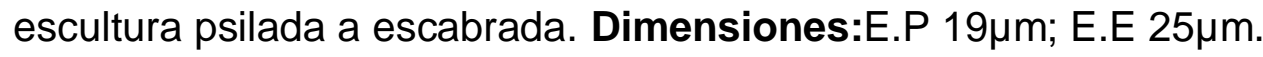

\subsection{Familia Arecaceae}

Tipo Bactris (Lámina 2, Fig.16-16a)

Descripción: Mónada, heteropolar, simétrica bilateral, ámbito elíptico; trichotomosulcado, sulco de $24 \mu \mathrm{m}$ de largo por $2 \mu \mathrm{m}$ de ancho, margen de $2 \mu \mathrm{m}$ de grosor; exina semitectada de $2 \mu \mathrm{m}$ de grosor, columelas conspicuas, escultura reticulada, con lúmenes y muroshomobrochados $\leq 1 \mu \mathrm{m}$ de forma angular. Dimensiones:E.P $32 \mu \mathrm{m}$; E.E $29 \mu \mathrm{m}$.

Tipo Geonomma (Lámina 2, Fig. 17)

Descripción: Mónada, heteropolar, simétrica bilateral, ámbito elíptico; trichotomosulcado; exina tectada de $2 \mu \mathrm{m}$ de grosor, columelasinconspicuas, escultura rugulada, rugulae distribuidas sobre toda la superficie del grano. Dimensiones:D.E $50 \mu \mathrm{m}$. 


\subsection{Familia Bromeliaceae}

Tipo Bromeliacea esp (Lámina 2, Fig. 18)

Descripción: Mónada, heteropolar, simetría bilateral, ámbito biconvexo; monosulcada, margen de $2 \mu \mathrm{m}$ de grosor; exina semitectada de $2.5 \mu \mathrm{m}$ de grosor, columelas conspicuas, escultura reticulada con lúmenes heterobrochados $\geq$ $2.5 \mu \mathrm{m}$ de grosor, disminuyendo hacia el contorno del grano. Dimensiones:E.P $60 \mu \mathrm{m} ; \mathrm{E} . \mathrm{E} 45 \mu \mathrm{m}$.

\subsection{Familia Cyperaceae}

Tipo Cyperaceae (Lámina 2, Fig. 19)

Descripción: Mónada, apolar, ámbito circular, tamaño del grano mediano; exina

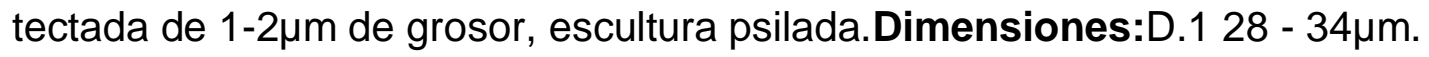

\subsection{Familia Poaceae}

Tipo Zea mays (Lámina 2, Fig. 20)

Descripción: Mónada, apolar, simétrica radial, ámbito circular, grano de gran tamaño;monoporado, poro de $3 \mu \mathrm{m}$ de largo por $8 \mu \mathrm{m}$ de ancho, anillo de $3 \mu \mathrm{m}$ de grosor; exina tectada de $3 \mu \mathrm{m}$ de grosor, escultura microreticulada.Dimensiones:D1: $82 \mu \mathrm{m}$.

Tipo Poaceae sp1 (Lámina 2, Fig. 21)

Descripción: Mónada, apolar, simétrica bilateral, ámbito circular, grano de tamaño medio; monoporado, poro de $2 \mu \mathrm{m}$ de largo por $5 \mu \mathrm{m}$ de ancho, anillo de $2 \mu \mathrm{m}$ de grosor con un margen semidentado; exina tectada de $2 \mu \mathrm{m}$ de grosor, escultura psilada a escabrada.Dimensiones:D1: $25 \mu \mathrm{m}$.

Tipo Poaceae sp2 (Lámina 2, Fig. 22)

Descripción: Mónada, apolar, simétrica radial, ámbitocircular, grano de tamaño medio; monoporado, poro de $5 \mu \mathrm{m}$ de largo por $7 \mu \mathrm{m}$ de ancho, anillo de $2 \mu \mathrm{m}$ de grosor; exina tectada de $2 \mu \mathrm{m}$ de grosor, escultura perforada.Dimensiones:D1: $42 \mu \mathrm{m}$.

\section{Eucotiledoneas}

\subsection{Familia Acanthaceae}

Acanthaceae (Lámina 2, Fig. 23-23a)

Descripción: Mónada, isopolar, simétrica radial, forma prolado ( $P / E$ 1.3); tricolpado, colpos de $17 \mu \mathrm{m}$ de largo por $2 \mu \mathrm{m}$ de ancho, con margen de $2 \mu \mathrm{m}$ de 
grosor; exina tectada de $3 \mu \mathrm{m}$ de grosor, columelas conspicuas, escultura escabrada a perforada. Dimensiones: E.P $22 \mu \mathrm{m}$; E.E $16 \mu \mathrm{m}$.

\subsection{Familia Amaranthaceae}

Tipo Amaranthus (Lámina 2, Fig. 24-24a)

Descripción: Mónada, apolar, asimétrica, ámbito elíptico, ámbito esferoidal; tamaño del grano mediano; periporado entre 18 a 22 poros circulares de $3 \mu \mathrm{m}$; exina semitectada de $3 \mu \mathrm{m}$ de grosor, escultura reticulada de lúmenes heterobrocados. Dimensiones: D1: $30 \mu \mathrm{m}$.

\subsection{Familia Anacardiaceae}

TipoAnacardiaceae (Lámina 2, Fig. 25-25a)

Descripción: Mónada, isopolar, simétrica radial, ámbito circular, subprolado (P/E 1.16), área polar mediana (I.A.P: 0.33), tamaño del grano pequeño; tricolporado, colpos estrechos de $17 \mu \mathrm{m}$ de largo por $2 \mu \mathrm{m}$ de ancho, poro lalongado de $2 \mu \mathrm{m}$ de largo por $4 \mu \mathrm{m}$ de ancho; exina tectada de $1 \mu \mathrm{m}$ de grosor, escultura estriada. Dimensiones: E.P $21 \mu \mathrm{m}$; E.E $18 \mu \mathrm{m}$; D.E $18 \mu \mathrm{m}$; L.A $6 \mu \mathrm{m}$.

\subsection{Familia Apocynaceae}

Tipo Lacmellea (Lámina 2, Fig. 26-26a)

Descripción: Mónada, isopolar, simétrica bilateral o radial, ámbito elíptico, forma prolado (P/E 1.33); diporado a triporado, poros de $3 \mu \mathrm{m}$ de largo por $4 \mu \mathrm{m}$ de ancho, de forma circular; exina $\geq 1 \mu \mathrm{m}$ de grosor, tectada y de escultura psilada. Dimensiones: E.P $12 \mu \mathrm{m}$; E.E $20 \mu \mathrm{m}$; D.E $14 \mu \mathrm{m}$.

\subsection{Familia Aquifoliaceae}

Tipo Ilex (Lámina 2, Fig. 27)

Descripción: Mónada, isopolar, simétrica radial, forma prolado (P/E 1.15); tricolporado, colpos de $17 \mu \mathrm{m}$ de largo por $3 \mu \mathrm{m}$ de ancho, constrictos hacia el ecuador; poros pequeños, lolongados de $2 \mu \mathrm{m}$ de largo por $3 \mu \mathrm{m}$ de ancho; exina intectada, escultura clavada, clavas de diferentes tamaños, variando entre $2-4 \mu \mathrm{m}$ de largo por $1 \mu \mathrm{m}$ de ancho. Dimensiones: E.P $22 \mu \mathrm{m}$; E.E $30 \mu \mathrm{m}$.

\subsection{Familia Asteraceae}

Tipo Ambrosia (Lámina 3, Fig. 28)

Descripción: Mónada, isopolar, simétrica radial, forma oblado ( $P / E$ 1.04); tricolporado, colpos de $17 \mu \mathrm{m}$ de largo por $3 \mu \mathrm{m}$ de ancho; poros pequeños, lolongados de $2 \mu \mathrm{m}$ de largo por $3 \mu \mathrm{m}$ de ancho; exina tectada de escultura 
equinada, equinas de forma triangular y ápices agudos. Dimensiones: E.P 22 $\mu$ m; E.E $21 \mu \mathrm{m}$.

Tipo Sonchus (Lámina 3, Fig. 29-29a)

Descripción: Mónada, isopolar, simétrica radial, forma esferoidal (P/E 1); exina semitectada de $4 \mu \mathrm{m}$ de grosor; escultura equino-fenestrada, muros de $4 \mu \mathrm{m}$ de grosor, lagunas rectangulares de $5 \mu \mathrm{m}$, espinas delgadas de $3 \mu \mathrm{m}$ de largo por $1 \mu \mathrm{m}$ de ancho, triangular y de ápices agudos. Dimensiones: D.E $25 \mu \mathrm{m}$.

TipoAcmella (Lámina 3, Fig. 30-30a)

Descripción: Mónada, isopolar, simétrica radial, forma subprolado ( $P / E$ 1.16); tricolporado, colpo de $11 \mu \mathrm{m}$ de largo por $1 \mu \mathrm{m}$ de ancho, poros lalongados de $1 \mu \mathrm{m}$ de largo por $2 \mu \mathrm{m}$ de ancho; exina tectada $\leq 1 \mu \mathrm{m}$ de grosor, escultura equinada, equinas de forma triangular y ápices agudos. Dimensiones: E.P $14 \mu \mathrm{m}$; E.E $12 \mu \mathrm{m}$.

Tipo Asteraceae sp1 (Lámina 3, Fig. 31)

Descripción: Mónada, isopolar, simétrica radial, forma oblado esferoidal ( $P / E$ 0.94); tricolporado, colpo de $10 \mu \mathrm{m}$ de largo por $2 \mu \mathrm{m}$ de ancho, poros lalongados; exina tectada $\leq 1 \mu \mathrm{m}$ de grosor, escultura equinada, equinas de forma triangular $y$

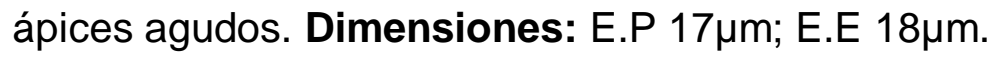

TipoAsteraceae sp2 (Lámina 3, Fig. 32)

Descripción: Mónada, isopolar, simétrica radial, forma prolado esferoidal (P/E 1.04); tricolporado, colpo de $12 \mu \mathrm{m}$ de largo por $1 \mu \mathrm{m}$ de ancho, poros lolongados; exina tectada de $4 \mu \mathrm{m}$ de grosor, escultura equinada, equinas de forma triangular y ápices agudos. Dimensiones: E.P 25 $\mu$ m; E.E $24 \mu \mathrm{m}$.

\subsection{Familia Betulaceae}

Tipo Alnus (Lámina 3, Fig. 33)

Descripción: Mónada, isopolar, simétrica radial, ámbito poligonal; estefanoporado $(3,4,5,6 \circ 7)$, poros circulares de $1 \mu \mathrm{m}$, anillo de $1 \mu \mathrm{m}$ de grosor, sobresaliente, poros unido por una banda 0 arco; exina tectada $<1 \mu \mathrm{m}$ de grosor, escultura escabrada a psilada. Dimensiones: D.E $29 \mu \mathrm{m}$.

\subsection{FamiliaBignoniaceae}

Tipo Tabebuia (Lámina 3, Fig. 34)

Descripción: Mónada, isopolar, simétrica radial, forma sub-prolada ( $\mathrm{P} / \mathrm{E}$ 1.3); tricolpado, colpos largos y estrechos de $29 \mu \mathrm{m}$ de largo por $3 \mu \mathrm{m}$ de ancho; exina 
semitectada de $3 \mu \mathrm{m}$ de grosor, de escultura reticulada, lúmenes homobrochados.

Dimensiones: E.P $38 \mu \mathrm{m}$; E.E $28 \mu \mathrm{m}$.

\subsection{FamiliaBegoniaceae}

Tipo Begonia (Lámina 3, Fig. 35)

Descripción: Mónada, isopolar, simétrica radial, forma perprolado ( $P / E$ 2); tricolporado, colpos de $20 \mu \mathrm{m}$ de largo por $1 \mu \mathrm{m}$ de ancho, costa de $1 \mu \mathrm{m}$ de grosor, poro lalongado y operculado; exina semitectada, escultura micro-reticulada, lúmenes homobrochados. Dimensiones: E.P $20 \mu \mathrm{m}$ E.E 10 $\mu \mathrm{m}$.

\subsection{Familia Cecropiaceae}

Tipo Cecropia (Lámina 3, Fig. 36)

Descripción: Mónada, isopolar, simétrica bilateral, forma subprolado (P/E 1.28); diporado, poros circulares de $1 \mu \mathrm{m}$, anulados; exina tectada $<1 \mu \mathrm{m}$ de grosor, escultura escabrada a psilada. Dimensiones: E.P $9 \mu \mathrm{m}$; E.E $7 \mu \mathrm{m}$.

\subsection{Familia Clethraceae}

Tipo Clethra (Lámina 3, Fig. 37)

Descripción: Mónada, isopolar, simétrica radial de ámbito circular, forma oblado esferoidal (P/E 0.92); tricolporado, colpos estrechos de $12 \mu \mathrm{m}$ de largo por $1 \mu \mathrm{m}$ de ancho, costa de $<1 \mu \mathrm{m}$ de grosor, poro lolongado y puede o no ser visible; exina tectada de $1 \mu \mathrm{m}$ de grosor, escultura psilada a escabrada. Dimensiones: E.P $12 \mu \mathrm{m}$; E.E $13 \mu \mathrm{m}$.

\subsection{Familia Cloranthaceae}

Tipo Hedyosmum (Lámina 3, Fig. 38)

Descripción: Mónada, isopolar, simétrica radial, ámbito circular, tamaño del grano mediano; inaberturado; exina intectada, escultura clavada, clavas de $1 \mu \mathrm{m}$ de largo por $<1 \mu \mathrm{m}$ de ancho, distribuidas densamente en la superficie del grano. Dimensiones: D1: 25-30 $\mathrm{mm}$.

\subsection{Familia Cucurbitaceae}

Tipo Melothriapendula (Lámina 3, Fig. 39-39a)

Descripción: Mónada, isopolar, simétrica radial, ámbito triangular obtusoconvexo, forma prolado esferoidal (P/E 1.02), área polar grande (I.A.P: 0.51), tamaño del grano mediano; tricolporado, colpos de $27 \mu \mathrm{m}$ de largo por $3 \mu \mathrm{m}$ de ancho, poro lolongado de $6 \mu \mathrm{m}$ de largo por $8 \mu \mathrm{m}$ de ancho; exina semitectada de $5 \mu \mathrm{m}$ de ancho, escultura reticulada, con lúmenes homobrochados y ovalados

Dimensiones: E.P $46 \mu \mathrm{m}$; E.E $45 \mu \mathrm{m}$; D.E $49 \mu \mathrm{m}$; L.A $25 \mu \mathrm{m}$. 


\subsection{Familia Cunoniaceae}

Tipo Weinmmania (Lámina 3, Fig. 40-40a)

Descripción: Mónada, isopolar, simétrica radial, forma subprolado(P/E 1.2); tricolporado, colpos de $9 \mu \mathrm{m}$ de largo por $1 \mu \mathrm{m}$ de ancho; exina semitectada de $1.2 \mu \mathrm{m}$ de grosor, escultura microreticulada, lumen homobrochado. Dimensiones: E.P $12 \mu \mathrm{m} ; \mathrm{E} . \mathrm{E} 10 \mu \mathrm{m}$.

\subsection{Familia Euphorbiaceae}

TipoAcalypha (Lámina 3, Fig. 41)

Descripción: Mónada, isopolar, simétrica radial, ámbito cuadrangular obtusorecto, en algunos casos más o menos circular, tamaño del grano pequeño; estephanocolporado, entre 4 a 5 colporos, brevicolpos únicamente visibles en 100X; exina tectada de $1.2 \mu \mathrm{m}$ de grosor, escultura psilada a ligeramente escabrada. Dimensiones: D.E22 $\mu \mathrm{m}$.

Tipo Faramea (Lámina 3, Fig. 42-42a)

Descripción: Mónada, isopolar, simétrica radial, ámbito triangular obtuso-convexo o cuadrangular, forma peroblado a oblado (P/E 1.5), tamaño del grano pequeño; estefanoporado, 3-4 poros circulares de $3 \mu \mathrm{m}$, vestibulum y aspide presentes; exina semitectada de $1 \mu \mathrm{m}$ de grosor, escultura reticulada con lúmenes

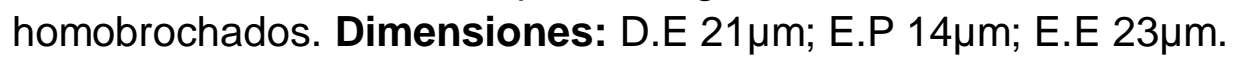

Tipo Ricinus communis (Lámina 3, Fig. 43-43a)

Descripción: Mónada, isopolar, simétrica radial, ámbito triangular obtusoconvexo, forma oblado esferoidal (P/E 0.94), área polar pequeña (I.A.P: 0.22), tamaño del grano mediano; tricolporado, colpos de $27 \mu \mathrm{m}$ de largo por $2.5 \mu \mathrm{m}$ de ancho,margen de $1 \mu \mathrm{m}$ de grosor, poro lolongado de $4 \mu \mathrm{m}$ de largo por $4.5 \mu \mathrm{m}$ de ancho; exinasemitectadade $3 \mu \mathrm{m}$ de grosor, escultura reticulada, lúmenes

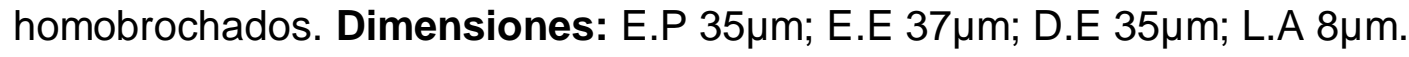

\subsection{Familia Ericaceae}

Tipo Bejaria (Lámina 4, Fig. 44-44a)

Descripción: Tétrada tetrahedral;Mónada,isopolar, simétrica radial, ámbito circular, tamaño del grano mediano, área polar grande (I.A.P: 0.63); brevitricolporados; exina tectada de $3.4 \mu \mathrm{m}$ de grosor, escultura rugulada.

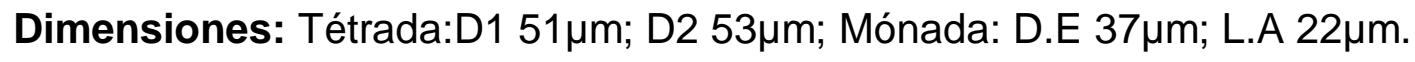

\subsection{Familia Fabaceae}

Tipo Dalea (Lámina 4, Fig. 45) 
Descripción: Mónada, isopolar, simétrica radial, forma prolado ( $P / E$ 1.9); tricolpado, colpos estrechos de $27 \mu \mathrm{m}$ de largo por $1 \mu \mathrm{m}$ de ancho; exina semitectada, escultura reticulada, lúmenes homobrochados, los cuales reducen su tamaño hacia el mesocolpo. Dimensiones: E.P $31 \mu \mathrm{m}$; E.E 16 $\mathrm{m}$.

\subsection{Familia Fagaceae}

Tipo Quercus (Lámina 4, Fig. 46)

Descripción: Mónada, isopolar, simétrica radial, forma prolado ( $\mathrm{P} / \mathrm{E}$ 1.75); tricolporado, colpos de $20 \mu \mathrm{m}$ de largo por $2 \mu \mathrm{m}$ de ancho, margen de $2 \mu \mathrm{m}$ de grosor, colpos constreñidos hacia el ecuador; exinatectadade $1.8 \mu \mathrm{m}$ de grosor,

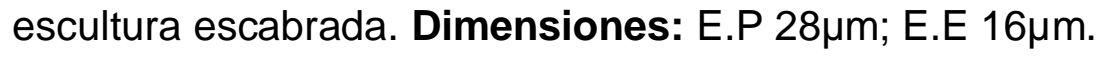

\subsection{Familia Gesneriaceae}

Tipo Besleria (Lámina 4, Fig. 47)

Descripción: Mónada, isopolar, simétrica radial, forma prolado ( $P / E$ 1.6); tricolpado, colpos estrechos de $21 \mu \mathrm{m}$ de largo por $2 \mu \mathrm{m}$ de ancho, margen de $1.2 \mu \mathrm{m}$ de grosor; exina semitectada de $3 \mu \mathrm{m}$ de grosor, escultura micro-reticulada, lúmenes homobrochados $<1 \mu \mathrm{m}$ de forma ovalada. Dimensiones: E.P $24 \mu \mathrm{m}$; E.E $15 \mu \mathrm{m}$.

\subsection{Familia Malpighiaceaeae}

Tipo Stigmaphyllon (Lámina 4, Fig. 48-48a)

Descripción: Mónada, apolar, ámbito circular, grano pequeño $(20 \mu \mathrm{m})$, periporado, 6 poros ovales de $3 \mu \mathrm{m}$, anillo entre $\leq 1 \mu \mathrm{m}$ de grosor; exina tectada de $3 \mu \mathrm{m}$ de grosor, escultura psilada. Dimensiones: D1: $20 \mu \mathrm{m}$.

\subsection{FamiliaMalvaceae}

Tipo Malvaceae (Lámina 4, Fig. 49)

Descripción: Mónada, apolar, ámbito circular, grano de gran tamaño; periporado, 12 poros de $3 \mu \mathrm{m}$ largo por $2 \mu \mathrm{m}$ de ancho; exina tectada de $5 \mu \mathrm{m}$ de grosor, columelas conspicuas, escultura equinada, equinas de $5 \mu \mathrm{m}$ de largo por $3 \mu \mathrm{m}$ de ancho, sobre toda la superficie del grano; los espacios entre las espinas presentan una superficie gemada. Dimensiones: D1: $70 \mu \mathrm{m}$.

\subsection{Familia Melastomataceae}

Tipo Melastomataceae (Lámina 4, Fig. 50-50a)

Descripción: Mónada, isopolar, simétrica radial, ámbito circular, forma suprolado (P/E 1.14), área polar muy pequeña (I.A.P: 0.15), tamaño del grano pequeño; heteroestefanocolporado, con 3 colporos y 3 pseudocolposde $12 \mu \mathrm{m}$ de largo por 
$2 \mu \mathrm{m}$ de ancho; exina tectada de $1 \mu \mathrm{m}$ de grosor, escultura psilada, escabrada y

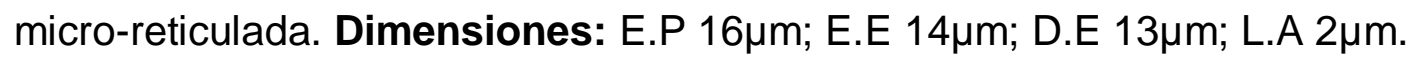

\subsection{FamiliaMimosaceae}

Tipo Mimosaceae (Lámina 4, Fig. 51-51a)

Descripción:Poliade de 14 a 16 granos simétricamente arreglados 3-3 o 3-4, forma elíptica a circular; grano aislado: apolar, asimétrico; inaberturado; exina tectada de $1 \mu \mathrm{m}$ de grosor, escultura psilada. Dimensiones: PoliadeD.1 60-50 $\mu \mathrm{m}$; Monada: $13-15 \mu \mathrm{m}$.

\subsection{Familia Moraceae}

Tipo Moraceae (Lámina 4, Fig. 52)

Descripción: Mónada, isopolar, simétrica radial, ámbito circular, forma suboblado (P/E 0.85); triporado, 3 poros de $1 \mu \mathrm{m}$; exina tectada de $1 \mu \mathrm{m}$ de grosor, escultura escabrada a micro-reticulada. Dimensiones: E.P $12 \mu \mathrm{m}$; E.E $14 \mu \mathrm{m}$; D.E $14 \mu \mathrm{m}$.

\subsection{Familia Muntigiaceae}

TipoMuntingia (Lámina 4, Fig. 53)

Descripción: Mónada, isopolar, simétrica radial, ámbito triangular obtusoconvexo, forma prolado (P/E 1.31), área polar mediana (I.A.P: 0.28), tamaño del grano pequeño; tricolporado, colpos estrechos de $16 \mu \mathrm{m}$ de largo por $1 \mu \mathrm{m}$ de ancho, poros lalongados de $2 \mu \mathrm{m}$ de largo por $4 \mu \mathrm{m}$ de ancho; exina semitectada de $\leq 3 \mu \mathrm{m}$ de grosor, escultura reticulada, con lúmenes homobrochados.

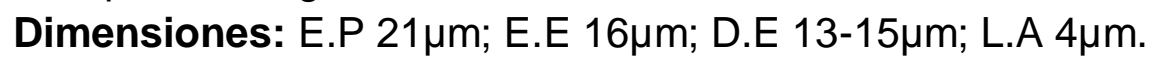

\subsection{Familia Myrcinaceae}

TipoArdisia (Lámina 4, Fig. 54-54a)

Descripción: Mónada, isopolar, simétrica radial, ámbito circular a elíptico, forma subprolado (P/E 1.18), área polar mediana (I.A.P: 0.30); tricolporado, colpos de $17 \mu \mathrm{m}$ de largo por $1 \mu \mathrm{m}$ de ancho; exina semitectada de escultura reticulada.

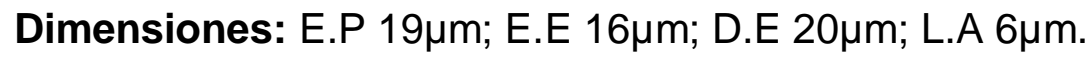

TipoMyrcine (Lámina 4, Fig. 55)

Descripción: Mónada, isopolar, simétrica radial, ámbito cuadrangular; tetracolporado, área polar mediana (I.A.P: 2.2); exina tectada de $3 \mu \mathrm{m}$ de grosor, escultura psilada a reticulada. Dimensiones: D.E $22 \mu \mathrm{m}$; L.A $10 \mu \mathrm{m}$.

\subsection{Familia Myrtaceae}

Tipo Myrtaceae (Lámina 4, Fig. 56) 
Descripción: Mónada, isopolar, simétrica radial, ámbito triangular obtusoconvexo; parasyncolpado; anillo presente de $3 \mu \mathrm{m}$ de grosor; exina tectada de $2 \mu \mathrm{m}$ de grosor, escultura psilada a escabrada. Dimensiones: D.E $28 \mu \mathrm{m}$.

\subsection{FamiliaPiperaceae}

Tipo Peperomia (Lámina 4, Fig. 57)

Descripción: Mónada, isopolar, simétrica bilateral, forma a subprolado (P/E 1.4); monocolpado, colpo de $7 \mu \mathrm{m}$ de largo por $1 \mu \mathrm{m}$ de ancho; exina tectada $\leq 1 \mu \mathrm{m}$ de grosor, escultura gemada. Dimensiones: E.P 10 $\mathrm{m}$; E.E $7 \mu \mathrm{m}$.

Tipo Piper (Lámina 4, Fig. 58)

Descripción: Mónada, isopolar, simétrica bilateral, forma subprolado (P/E 1.4); monocolpado, colpo de $8 \mu \mathrm{m}$ de largo por $2 \mu \mathrm{m}$ de ancho; exina tectada $\leq 1 \mu \mathrm{m}$ de

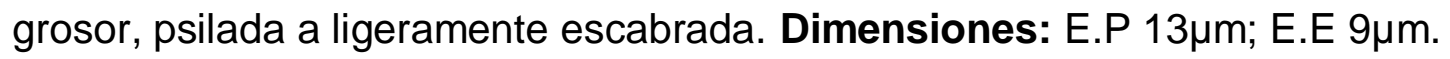

\subsection{Familia Rosaceae}

Tipo Rosaceae (Lámina 4, Fig. 59)

Descripción: Mónada, isopolar, simétrica radial, ámbito triangular obtusoconvexo, forma prolado esferoidal (P/E 1.14), área polar mediana (I.A.P: 0.44), tamaño del grano mediano; tricolporado, colpos de $20 \mu \mathrm{m}$ de largo por $\geq 3 \mu \mathrm{m}$ de ancho, poro lalongado de $2 \mu \mathrm{m}$ de largo por $1.6 \mu \mathrm{m}$ de ancho; exina tectada $\geq 1.6 \mu \mathrm{m}$ de grosor, escultura psilada a escabrada. Dimensiones: E.P $27 \mu \mathrm{m}$; E.E $21 \mu \mathrm{m}$; D.E $25 \mu \mathrm{m} ;$ L.A $11 \mu \mathrm{m}$.

\subsection{Familia Rubiaceae}

Tipo Borreria (Lámina 4, Fig. 60)

Descripción: Mónada, isopolar, simétrica radial, ámbito circular, forma circular (P/E 1), área polar grande (I.A.P: 0.52), tamaño del grano entre pequeño a mediano; estefanocolporado, 9 colporos, brevicolpos de $13 \mu \mathrm{m}$ de largo por $2 \mu \mathrm{m}$ de ancho, poros pequeños y circulares de $1 \mu \mathrm{m}$; exina semitectada de $3 \mu \mathrm{m}$ de grosor, escultura reticulada, con lúmenes homobrochados. Dimensiones: E.P $25 \mu \mathrm{m}$; E.E $25 \mu \mathrm{m}$; D.E $25 \mu \mathrm{m}$; L.A $13 \mu \mathrm{m}$.

Tipo Rubiaceae (Lámina 4, Fig. 61)

Descripción: Mónada, isopolar, simétrica radial, ámbito triangular obtusoconvexo, forma prolado esferoidal (P/E 1.2), área polar mediana (I.A.P: 0.28), tamaño del grano pequeño; tricolporado, colposestrechos de $15 \mu \mathrm{m}$ de largo por $2 \mu \mathrm{m}$ de ancho, poro lalongado de $1 \mu \mathrm{m}$ de largo por $3 \mu \mathrm{m}$ de ancho; exina 
semitectada de $3 \mu \mathrm{m}$ de grosor, escultura reticulada, con lúmenes homobrochados. Dimensiones: E.P $24 \mu \mathrm{m}$; E.E $20 \mu \mathrm{m}$; D.E $21 \mu \mathrm{m}$; L.A $6 \mu \mathrm{m}$.

\subsection{Familia Salicaceae}

Tipo Casearia (Lámina 4, Fig. 62)

Descripción: Mónada, isopolar, simétrica radial, ámbito circular, forma oblado esferoidal (P/E 0.93), área polar mediana (I.A.P: 0.26), tamaño del grano pequeño; estefanocolporado, 4 colporos de $12 \mu \mathrm{m}$ de largo por $2 \mu \mathrm{m}$ de ancho, poros circulares de $2 \mu \mathrm{m}$; exina tectada de $2 \mu \mathrm{m}$ de grosor, escultura psilada. Dimensiones: E.P $15 \mu \mathrm{m}$; E.E $16 \mu \mathrm{m}$; D.E $15 \mu \mathrm{m}$; L.A $4 \mu \mathrm{m}$.

\subsection{Familia Solanaceae}

Tipo Solanun (Lámina 4, Fig. 63)

Descripción: Mónada, isopolar, simétrica radial, forma subprolado (P/E 1.17); tricolporado, colpos de $16 \mu \mathrm{m}$ de largo por $1 \mu \mathrm{m}$ de ancho, costa de $2 \mu \mathrm{m}$ de grosor, poros lalongados de $4 \mu \mathrm{m}$ de largo por $7 \mu \mathrm{m}$ de ancho, formando un endocingulum; exina tectada de $2 \mu \mathrm{m}$ de grosor, escultura psilada, escabrada a perforada.

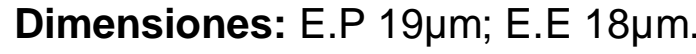

\section{Consideraciones finales}

Los resultados obtenidos en este estudio, son fundamentales para incrementar y contribuir al conocimiento palinológico del Cuaternario en la región del Cauca Medio. Los resultados muestran que la preservación de los granos de polen en suelos volcánicos es alta, la diversidad y buena preservación. Un total de 62 especies fueron descritas dentro de las cuales se encuentran representantes de clases Pteridophytoscon 11 especies de las familias Sellaginelaceae, Dryptoteridaceae, Polypodiaceae, Cyatheaceae, Dicksoniaceae, Lycopodiaceae y Pteridaceae, siendo la familia con mayor número de especies Polypodiaceae y Cyatheaceae.

Dentro de las plantas con semillas se encontraron 51 especies distribuidas en 1 Pinopsida, 50Angiospermas.Dentro de las Angiospermas se establecieron varias familias de Monocotiledoneas siendo la más representativa fue la Poaceae; mientras que dentro de las Eucotiledoneas las Familias Asteraceae, Euphorbiaceae y Rubiaceae fueron las más abundantes.

Por otra parte, es importante resaltar que dentro de los granos encontrados se hallaron varias especies con posible uso antrópicocomo Solanaceae y Poaceae. A 
la familia Solanaceae pertenece el pepino llorón (Solanumcaripense) y el lulo (Solanumquitoense), mientras que a la familia Poaceae se encuentra el maíz (Zea mays), una planta domesticada que depende claramente del ser humano. Un hallazgo importante es la aparición de los granos de polen de Amaranthussp., el cual ha sido descrito como una especie de alto valor nutritivo (DULL, 2007) y registrado en el Porce Medio (CASTILLO y ACEITUNO, 2006) como un taxón posiblemente cultivado. Aunque es importante tener en cuenta que en muchos casos especies de este género mantienen una clara preferencia hacia lugares que han sido perturbados y pueden ser encontradas como plantas adventicias al maíz (DULL, 2007).

En la cuenca del río San Eugenio, los suelos formados a partir de la sedimentación de cenizas volcánicas presentan una concentración polínica suficiente para llevar a cabo un estudio palinológico detallado, en una zona que carece de cuencas sedimentarias para el depósito y conservación de polen. Sin embargo, el alcance de los análisis palinológicos en sedimentos volcánicos es limitado en cuanto a los cambios a interpretaciones climáticas (MERCADO y ACEITUNO, en prep.), ya que estos sedimentos solo captan el polen de la vegetación alrededor del punto muestreado. Por consiguiente, las inferencias climáticas realizadas a partir de sedimentos volcánicos tienen más implicaciones a nivel local que regional, siendo necesario soportar y comparar los cambios climáticos con registros de polen regionales (MERCADO y ACEITUNO, en prep.).

\section{Agradecimientos}

El autor expresa sus agradecimientos a Ramiro Fonnegra por la revisión del manuscrito y al Comité para el Desarrollo de la Investigación (CODI) de la Universidad de Antioquia por el financiamiento al proyecto, al posgrado en biología y a la Universidad de Antioquia. 
Rev. Colombiana cienc. Anim. 4(2):410-434,2012

ORIGINAL
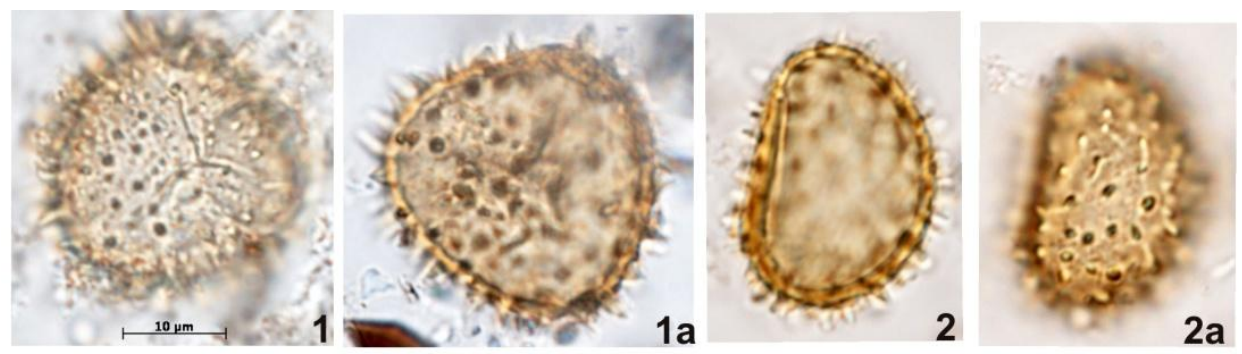

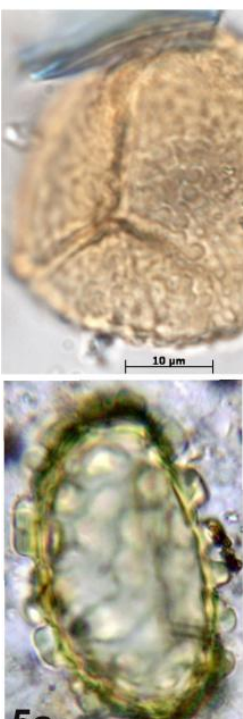

$5 a$
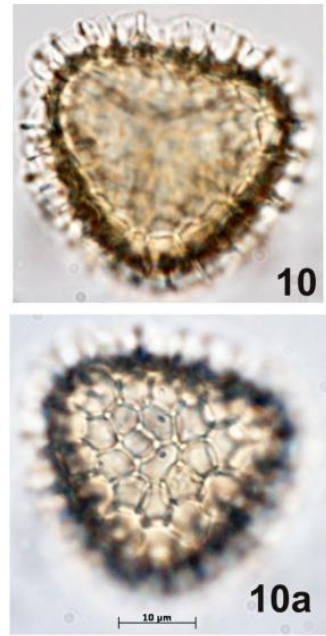
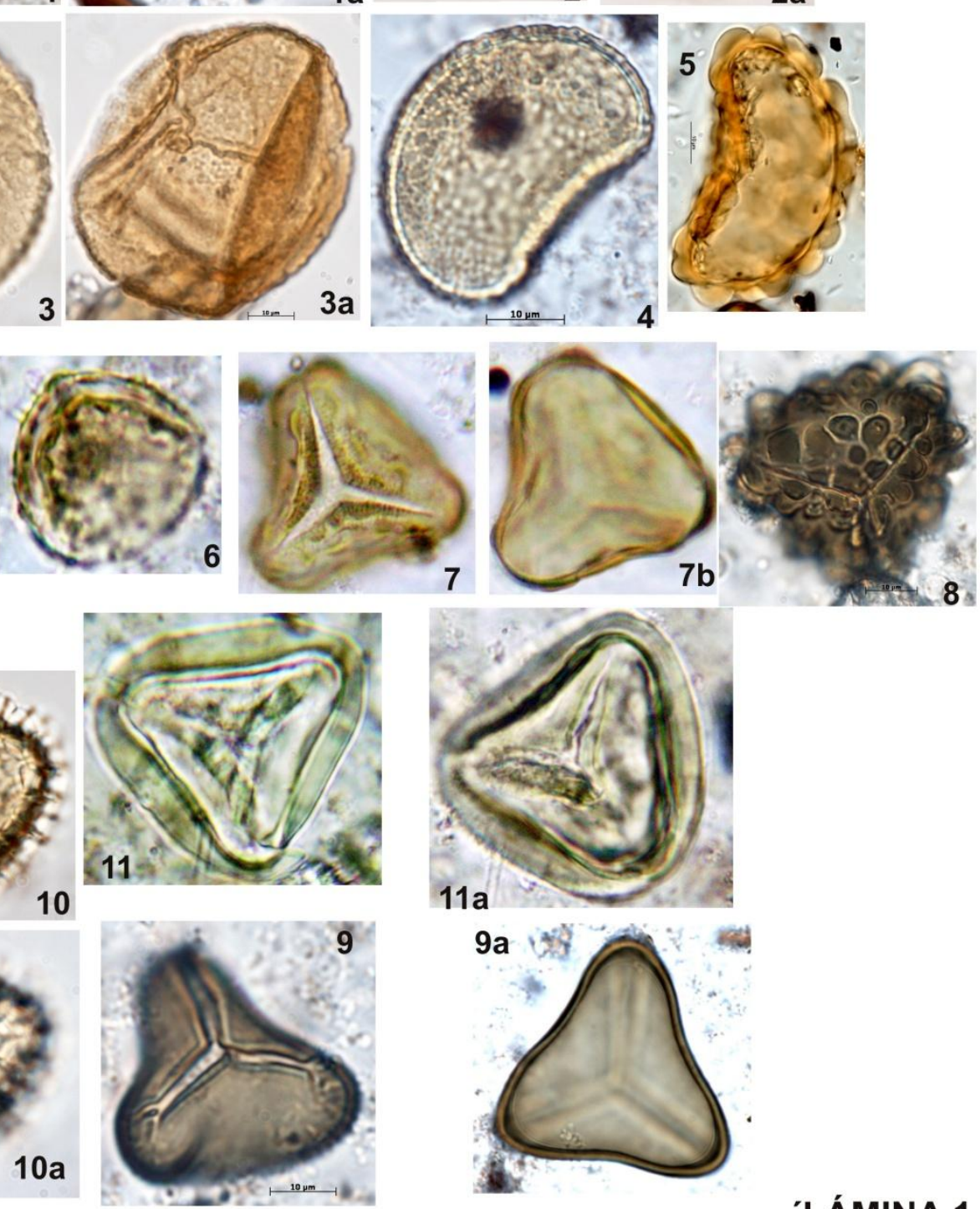

\section{'LÁMINA 1}

Lámina 1.Selaginella1-1a; Ctenitis 2-2a; Grammitis3-3a; Microgramma4; Polypodiaceae 5-5a; Monoleteperinado 6; Cyathea sp1 7-7a; Cyathea sp2 8; Dicksonia9-9a; Lycopodium10-10a; Jamesonia11-11a. 

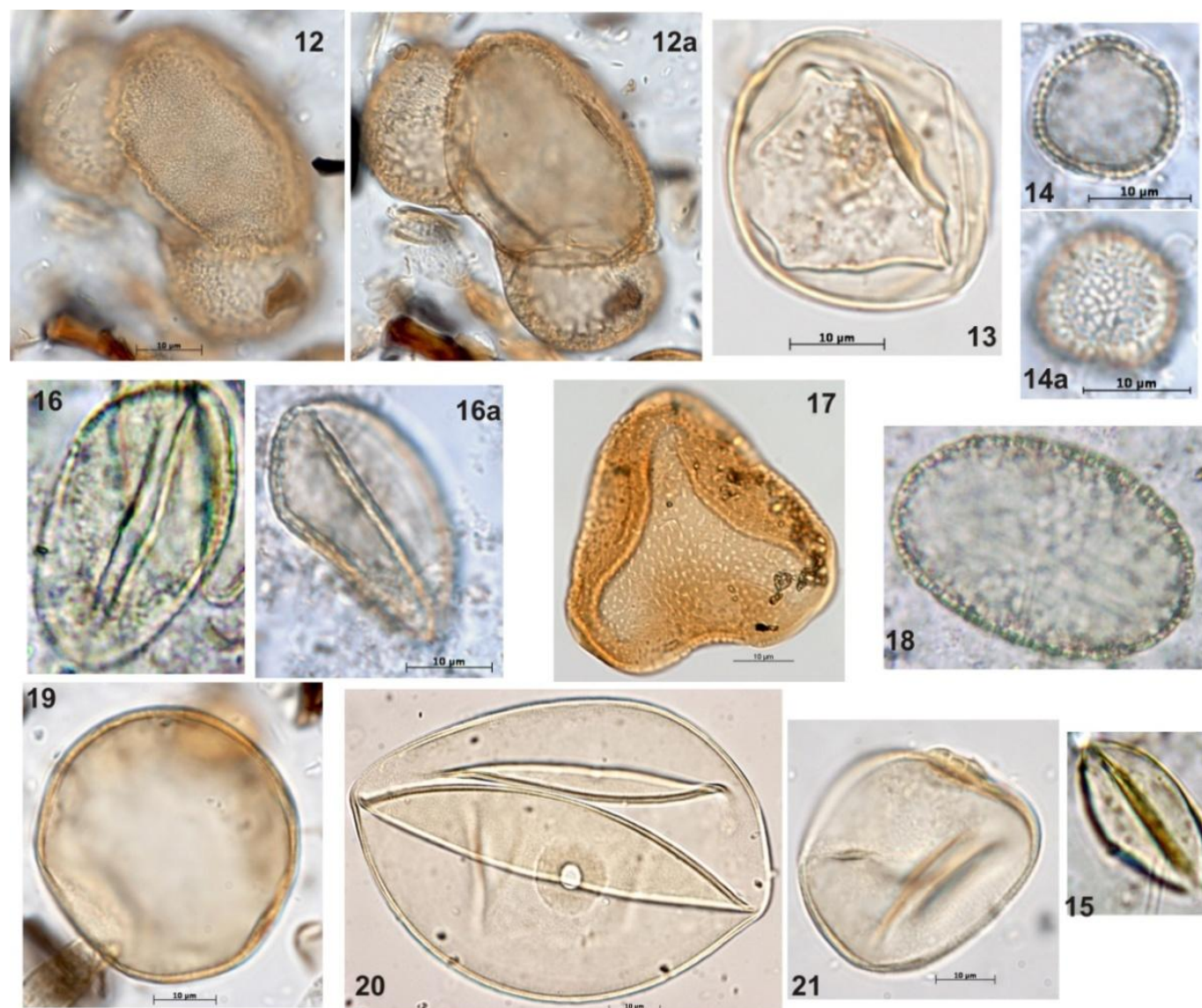

17
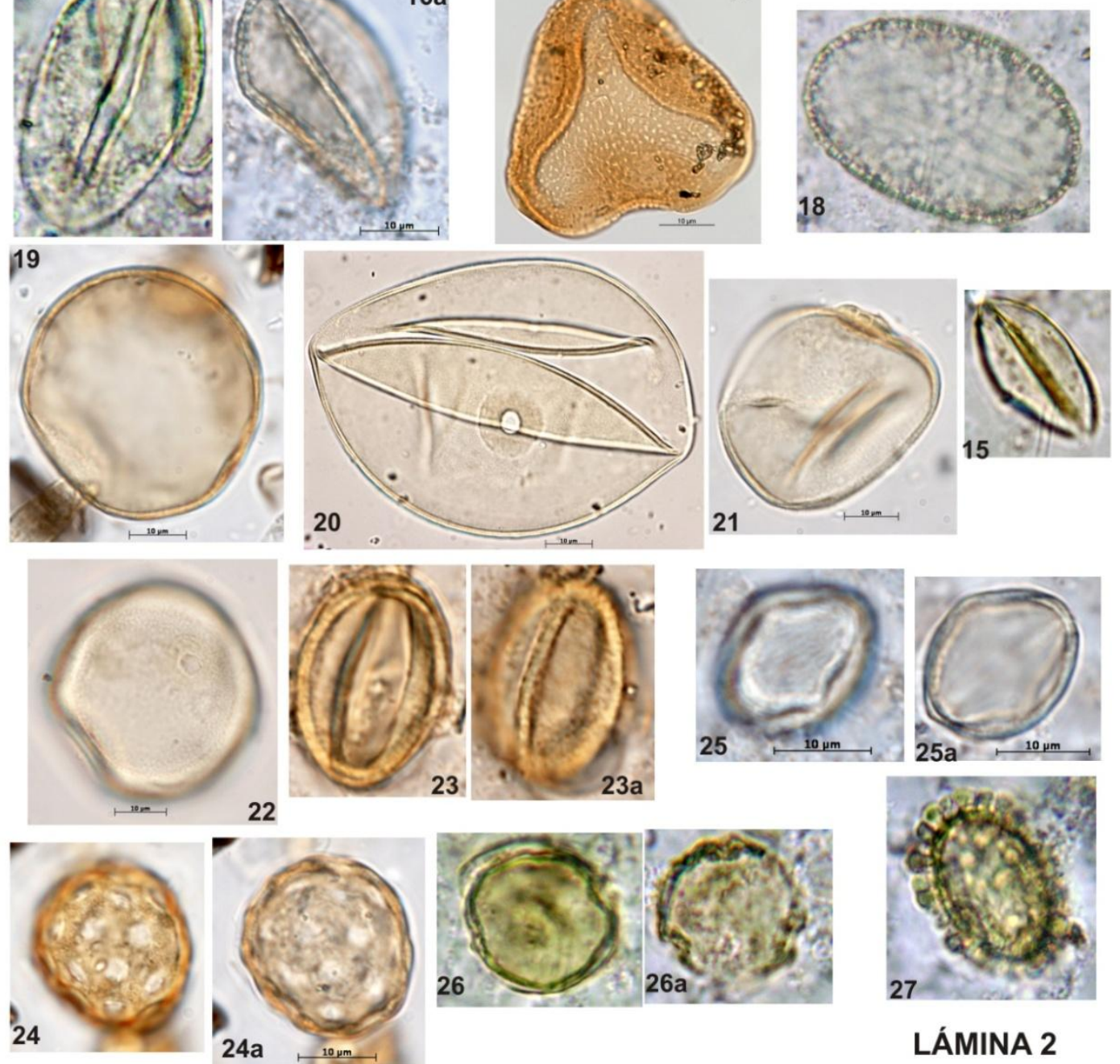

LÁMINA 2

Lámina 2.Podocarpus 12-12a; Araceae 13; Anthurium14-14a; Philodendrum15; Bactris 16-16a; Geonomma17; Bromeliaceaesp18; Cyperaceae 19; Zea mays20; Poaceae sp1 21; Poaceae sp2 22. 

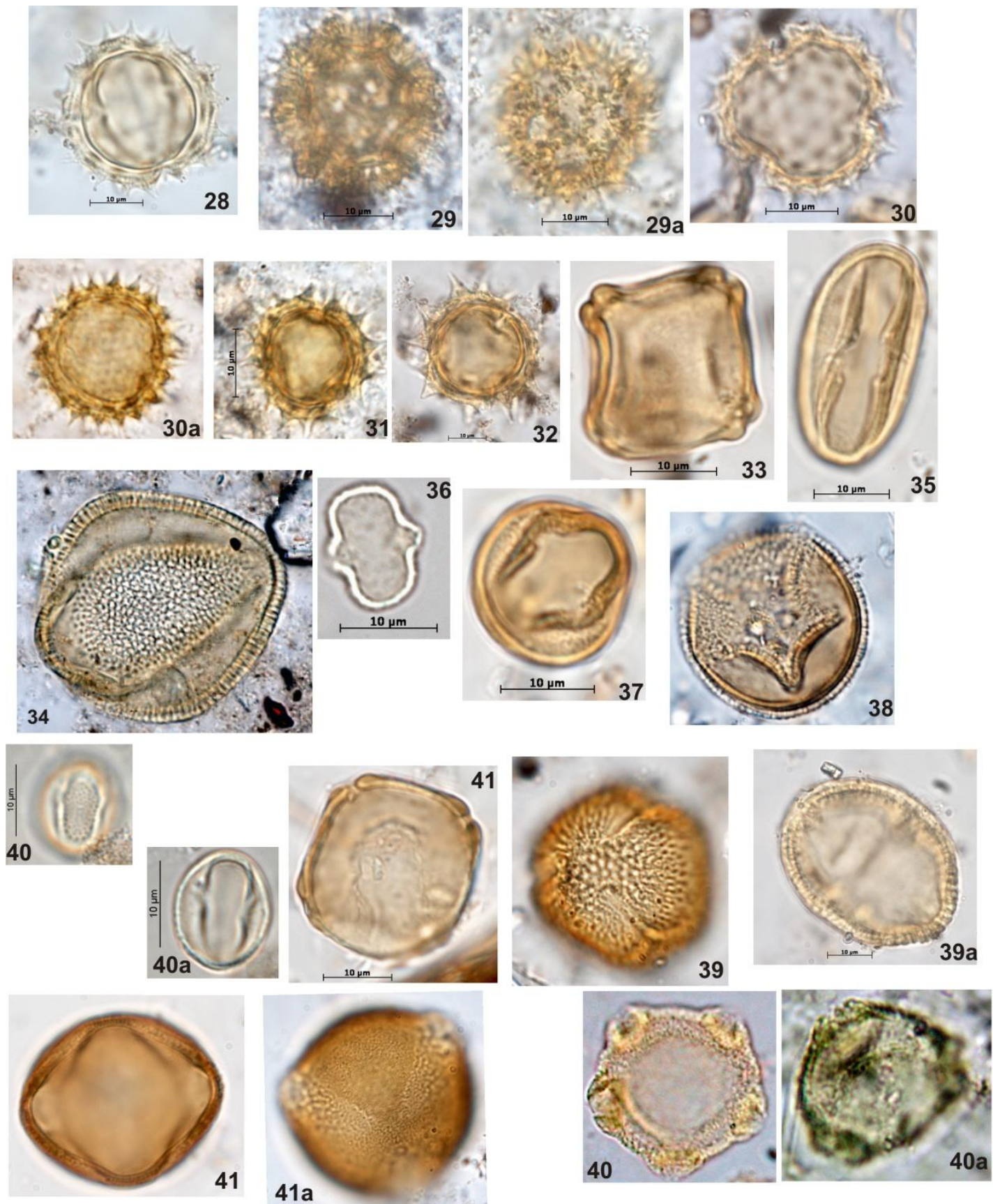

LÁMINA 3

Lámina 3: Ambrosia 28; Sonchus29-29a; Acmella30-30a; Asteraceae sp1 31; Asteraceae sp2 32; Alnus 33; Tabebuia 34; Begonia 35; Cecropia 36; Clethra37; Hedyosmum 38; Melothriapendula3939a; Weinmmania40-40a; Acalypha41; Faramea42-42a; Ricinuscommunis43-43a. 

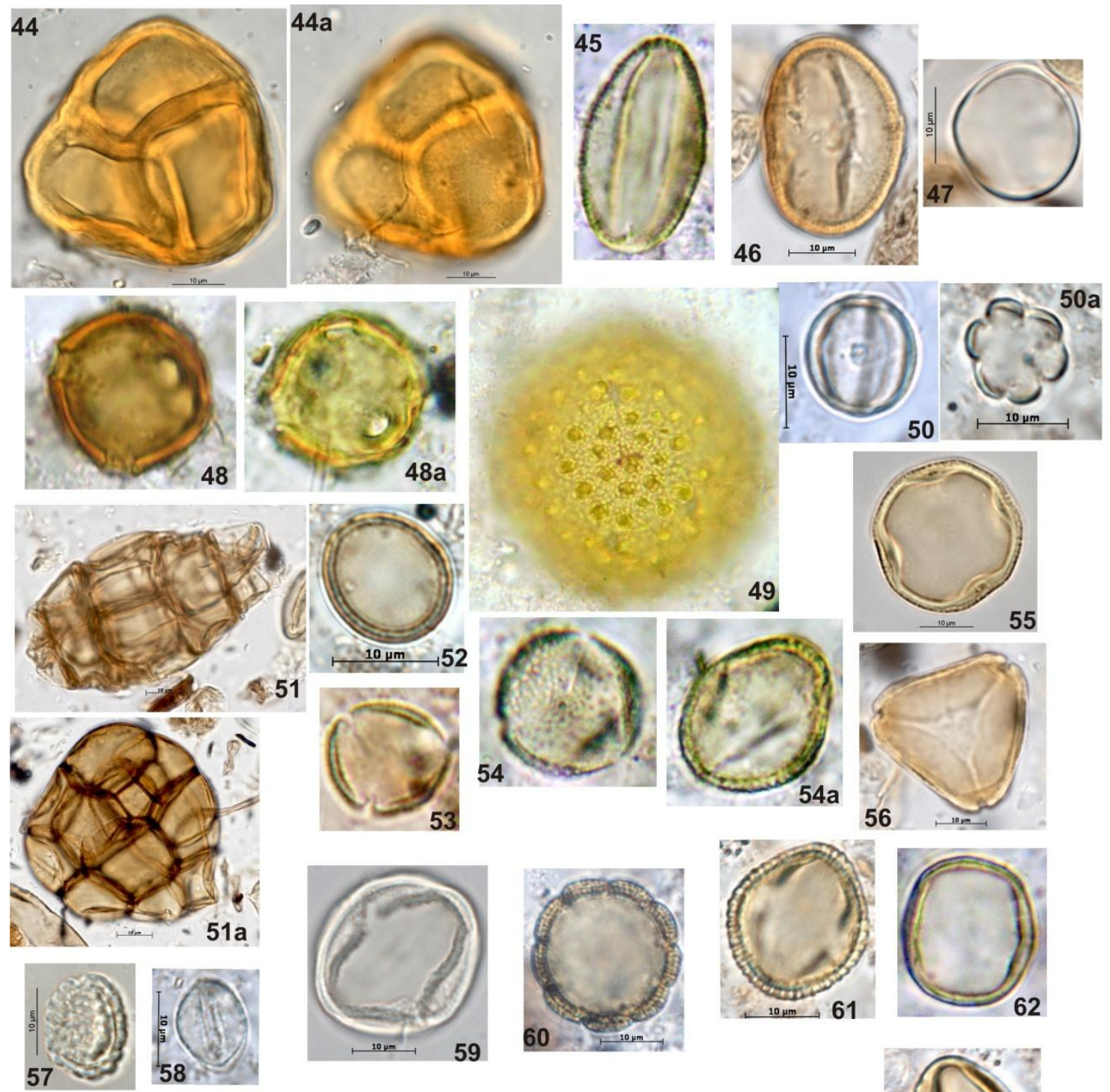

55
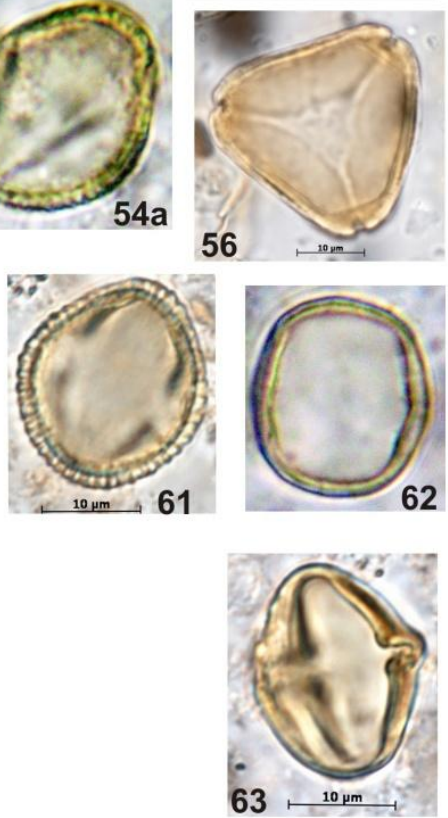

\section{LÁMINA 4}

Lámina 4. Bejaria44-44a; Dalea 45; Quercus46; Besleria47; Stigmaphyllon48-48a; Malvaceae 4949a; Melastomataceae50-50a; Mimosaceae 51-51a; Moraceae 52; Muntingia53; Ardisia54-54a; Myrcine55; Myrtaceae56; Peperomia57; Piper58; Rosaceae59; Borreria60; Rubiaceae61; Casearia 62; Solanun 63. 


\section{Referencias}

ACEITUNO F.J.; CASTILLO N. 2005. Mobility strategies in Colombia's middle mountain range between the early and middle Holocene. Before Farming 2 (2):117.

ACEITUNO F.J.; LOAIZA N. 2006. Ecología histórica en sociedades de pequeña escala Págs. 21-25 en F.J. Aceituno; N. Loaiza (ed.). Domesticación del bosque en el Cauca medio colombiano entre el Pleistoceno final y el Holoceno medio. Universidad de Antioquia y ICANH, Medellin

ACEITUNO F.J.; LOAIZA N. 2007. Domesticación del bosque en el Cauca medio colombiano entre el Pleistoceno final y el Holoceno medio: British Archaeological Reports. International Series, 1654. Oxford, Archaeopress.

BERRÍO J.C.; BOOM A.; BOTERO P.J.; HERRERA L.F.; HOOGHIEMSTRA H.; ROMERO F.; SARMIENTO G. 2001. Multi-disciplinary evidence of the Holocene history of a cultivated floodplain area in the wetlands of northern Colombia. Vegetation history and archaeobotany 10(3):161-174.

CASCAREDO A.C.; KLAUDIA O.; LOURDES C.H.; ETCHEVERS J.D.; CLAUDIA H.M. 2001. Estimación de alofano y su relación con otros parámetros químicos en andisoles de montaña del volcán Cofre de Perote. Terra 19(2):205-216.

CASTILLO N.; ACEITUNO F.J. 2006. El bosque domesticado, el bosque cultivado:un proceso milenario en el valle medio del río Porce en el Noroccidente Colombiano. Latin American Antiquity 17(4):1-18.

CORTÉS A.N. 1978. Los suelos en Colombia y su aptitud de uso. Colombia Geográfica 6(1):19-30.

DULL R.A. 2007. Evidence for forest clearance, agriculture, and human-Induced erosion in precolumbian El Salvador. Annals of the Association of American Geographers 97 (1): 127-141.

FAEGRI K.; IVERSEN J. 1975. Textbook of pollen analysis. Munksgaard, Copenhagen. 
HERRERA L.F.; URREGO L.E. 1996. Atlas de polen de plantas útiles y cultivadas de la Amazonia colombiana (Pollen atlas of useful and cultivated plants in the Colombian Amazon region). Estudios en la Amazonia Colombiana, XI: 462 pp. Tropenbos-Colombia, Bogotá (also published in 'The Quaternary of Colombia', 24 (ed. H. Hooghiemstra), Amsterdam).

HOOGHIEMSTRA H. 1984. Vegetational and climatic history of the high plain of Bogotá, Colombia: a continuous record of the last 3,5 million years. The quaternary of Colombia 79368.

HOOGHIEMSTRA H.; VAN DER HAMMEN T. 2004. Quaternary Ice-Age dynamics in the Colombian Andes: developing an understanding of our legacy. Philosophical Transactions of the Royal Society B: Biological Sciences 359(1442):173-181.

HOOGHIEMSTRA H.; WIJNINGA V.M.; CLEEF A.M. 2006. The paleobotanical record of Colombia: implications for biogeography and biodiversity. Annals of the Missouri Botanical Garden 93:297-324.

JARAMILLO D.F.; PARRA L.N.; GONZÁLEZ L.H. 1994. El recurso suelo en Colombia: distribución y evaluación. Medellín: ICNE. Universidad Nacional de Colombia.

MERCADO-GÓMEZ J. 2007. Polen de la clase Magnoliopsida para el VolcánPamplona (Norte de Santander - Colombia). Departamento de biología, vol. Facultad de ciencias básicas. Pamplona: Universidad de Pamplona, p. 145.

MERCADO-GÓMEZ J.; SOLANO L.; SANCHEZ L. 2007. Morfología polínica de especies pertenecientes a 5 géneros de Melastomataceae para Pamplona Colombia. Bistua 5(1):71-86.

MERCADO-GÓMEZ J.D.; JIMÉNEZ-BULLA L.C.; SÁNCHEZ-MONTAÑO L.R. 2011. Polen de las Magnoliopsida en el Volcán -Pamplona (Colombia) I: familias Apiaceae, Asteraceae, Cunoniaceae, Ericaceae, Fabaceae y Gentianaceae. Caldasia 33(2):619-635.

PIPERNO D.R.; PEARSALL D.M. 1998. The Origins of Agriculture in the Lowland Neotropics. New York. 
PUNT W.; BLACKMORE S.; NILSSON S.; THOMAS A.L. 1994. Glossary of pollen and spore terminology. Utrech: LPP Foundation. (LPP contributions series $\mathrm{N}^{\circ} 1$ ).

ROUBIK D.W.; MORENO J.E. 1991. Pollen and spores of Barro Colorado Island: Missouri Botanical Garden, 36, 270 pp.

SALOMONS J.B. 1986. Paleoecology of volcanic soils in the Colombian Central Cordillera (Parque Nacional Nautaral de los Nevados). The quaternary of Colombia 13:18-211.

WILLE M.; HOOGHIEMSTRA H.; BEHLING H.; VAN DER BORG K.; NEGRET A.J. 2001. Environmental change in the Colombian subandean forest belt from 8 pollen records: the last $50 \mathrm{kyr}$. Vegetation history and archaeobotany 10(2):61-77.

WILLE M.; NEGRET J.A.; HOOGHIEMSTRA H. 2000. Paleoenvironmental history of the Popayán area since 27000 yr BP at Timbio, Southern Colombia 109(1):4563.

ZARATE M.; NEME G.; GIL A. 2005. Mid-Holocene Paleoenvironments and human occupation in Southern South America. Quaternary International 132(1):13. 